\section{Physics by post}

\author{
Stephen G. Brush
}

The Correspondence between SIr George Gabriel Stokes and Sir William Thomson, Baron Kelvin of Largs, Vol. 1 \& II. Edited by David B. Wilson. Cambridge University Press: 1990. Pp. 783. £125, \$195.

IMAGINE that you could eavesdrop on the conversation of two eminent scientists once a month over a period of 55 years. Forget scandalous gossip and petty academic conspiracies - what you hear is mostly serious discussion of technical problems, interspersed with administrative trivia, news of family and colleagues, and evaluations of articles submitted for publication. These men are obsessed with science - doing research and running the institutions that nourish it. Personalities are a poor third.

Reading the letters of articulate scientific correspondents provides a feeling for how science works that is nowadays difficult for nonscientists to obtain without sitting in a laboratory for months. It also offers modern scientists an opportunity to compare their own workaholic life style with that of an earlier period.

William Thomson (1824-1907), known to posterity as Lord Kelvin, was the most famous British physicist of his day, recently the subject of a massive prizewinning biography by Crosbie Smith and Norton Wise (Energy and Empire, Cambridge University Press, 1989). His achievements, though now regarded as less profound than those of James Clerk Maxwell, were so numerous that at least some of them will certainly be known to any reader of Nature with a background in physical science.

George Gabriel Stokes (1819-1903) made his mark early on with important papers on hydrodynamics and optics but then devoted most of his energy in later years to administering the Royal Society and discharging his duties as Lucasian Professor at Cambridge.

Anything Stokes and Kelvin said to each other about science would have some historical value; a collection of 654 letters is a most magnificent treasure! As the editor notes, their correspondence is "easily the most extensive extant between two major Victorian physicists" and its publication is an important service to the history of science.

David B. Wilson's edition of the Stokes-Kelvin correspondence will be most useful to scholars who already know something about nineteenth-century physics, and about the careers of the two correspondents. He has previously published a short monograph, Kelvin and Stokes (Hilger, 1987), which amplifies the 47-page introduction to the Correspondence. His notes on the letters identify persons, events and publications mentioned, but do not attempt to elucidate the physics and mathematics. As Wilson admits, he follows current fashion among historians of science in concentrating instead on "institutional and oftenspeculative intellectual matters". I regard this as an act of generosity: he has done an enormous amount of work in making this primary source material easily available to other scholars, who can now (if they have the appropriate technical expertise) have

\section{IMAGE UNAVAILABLE FOR COPYRIGHT REASONS}

Sir George Gabriel Stokes (1819-1903)

the fun of discovering how it fits into and illuminates nineteenth-century science.

According to Wilson, the most significant thing we learn about scientific ideas from these letters is the change in Kelvin's views on the relation of ether to matter. Before the mid 1860s, he assumed that the Earth's atmosphere merged continuously into the interstellar medium that propagates light, so that air and ether are qualitatively the same thing. Stokes disagreed. Then, after being introduced to the kinetic theory of gases developed by Rudolf Clausius and Maxwell, Kelvin found that he could estimate the actual size of an atom, which turned out to be much bigger than he had previously thought. He therefore considered air a 'coarse' medium (large atoms with lots of space between them), and ether a very fine-grained medium. About this time (just before 1870) Kelvin became interested in the vortex atom theory - atoms might be described as stable vortex motions in a continuous (or at least much finergrained) fluid, the latter perhaps to be identified as the ether.

What seems remarkable to me is how little discussion of atoms and kinetic theory one finds in this correspondence. Most of the letters deal with macrophysics - electricity, magnetism, optics, hydrodynamics, elasticity, acoustics. Aside from the estimates of atomic sizes and discussions of the vortex atom, the index lists only three references in the letters to atoms and two other references to the dynamical or kinetic theory of gases. (To these could be added Kelvin's report of 15 June 1878 on Maxwell's kinetic theory of rarefied gases, not included in Wilson's edition because it was not addressed personally to Stokes.) Maxwell's striking result that the viscosity of a gas is independent of density, contrary to what Stokes and other experts on fluid flow had assumed, provided the first major triumph for his kinetic theory. Maxwell had written to Stokes on 30 May 1859 in the expectation that Stokes could give him an experimental refutation of this theoretical prediction. Indeed, Stokes and Kelvin had mentioned such experiments during the 1850s (letters 97-99, 110). But Maxwell's theory forced Stokes to reinterpret the experimental data (letters 209, 210, 473). Moreover, Maxwell's meanfree-path formula for gas viscosity provided part of the basis for Josef Loschmidt's and Kelvin's estimates of atomic diameters. Yet nowhere in the correspondence do we find any hint of the inference that twentiethcentury philosophers of science lead us to expect: because the kinetic theory successfully predicted a previously unknown empirical fact and stimulated fruitful research on gases, it was readily adopted as a working hypothesis. Instead, Kelvin and Stokes devoted much energy in their last years to arguing about the possibility of discontinuous motion in a continuous fluid.

For the modern reader, one of the most fascinating parts of the correspondence is the series of letters dated from 21 November 1896 to 19 April 1897 . Here we have a marvellous juxtaposition of the old and the new: classical hydrodynamics used to explain how oil calms troubled waters, and speculations about the nature of radioactivity whose effects Kelvin called "more like magic than anything I have ever seen or heard of in Science".

The editor and the publisher have produced an excellent book (bound in two handy volumes) which will be of great value for all future studies of nineteenthcentury physical science. And the then future Lord Kelvin, aged 22, has a warning that should be passed on to those lecturing on any subject at any time: "Yesterday I gave the introductory lecture, which was rather a failure as I had it all written, and I read it very fast".

Stephen G. Brush is in the Department of History and Institute for Physical Science and Technology, University of Maryland, College Park, Maryland 20742, USA. 